\title{
Retinal detachment due to paravascular abnormalities-associated breaks in highly myopic eyes
}

\author{
Yi-Ting Hsieh ${ }^{1} \cdot$ Chung-May Yang ${ }^{1,2}$
}

Received: 9 May 2018 / Revised: 12 August 2018 / Accepted: 26 August 2018 / Published online: 1 November 2018

(c) The Royal College of Ophthalmologists 2018

\begin{abstract}
Purpose To describe the characteristics of a novel type of retinal detachment (RD) due to paravascular abnormalities (PVAs) associated breaks in highly myopic eyes.

Methods We retrospective recruited eight eyes with RD due to PVA-associated breaks. The clinical features and surgical results were evaluated.

Results All eyes were highly myopic with a mean axial length of $31.31 \pm 2.86 \mathrm{~mm}(27.51-36.52 \mathrm{~mm})$. Two of the eight eyes had multiple paravascular retinal breaks associated with PVAs, four had a single paravascular break with PVAs nearby, and two had multiple paravascular breaks along the same vessels. All breaks were round or oval-shaped, and only three eyes (37.5\%) had their breaks located over patchy chorioretinal atrophy. Two of the eight eyes had the breaks outside the arcades, five had their breaks within the arcades, and one had a break within the arcade as well as breaks outside the arcade. Vitrectomy and epiretinal and internal limiting membrane peeling were performed in all cases, with inverted or free internal limiting membrane flap insertions for the perifoveal breaks and endolaser for the more peripheral breaks. All eyes had retinal reattachment after one single operation.

Conclusion RD due to PVA-associated breaks represented a distinct entity. PVAs and the associated breaks should be searched in RD without macular hole or obvious breaks in highly myopic eyes. It may be treated as RD caused by peripheral breaks or as macular hole associated RD depending on the location of the breaks.
\end{abstract}

\section{Introduction}

Paravascular abnormalities (PVAs) most frequently occur in eyes with high myopia or with epiretinal membrane (ERM). In 2008, Shimada et al. [1] first published their study on PVAs in eyes with high myopia and described the lesions including retinal cysts, microfolds, lamellar retinal hole, and retinoschisis including detachment of internal limiting membrane (ILM) along the retinal vessels or between two nearby vessels. They found vitreoretinal traction along the vessels next to the PVA lesions in almost all cases, and PVAs were most often present along the retinal vessel arcade. Li et al. [2] reported that $82.9 \%$ of 152 highly

Chung-May Yang

chungmay@ntu.edu.tw

1 Department of Ophthalmology, National Taiwan University Hospital, Taipei, Taiwan

2 College of Medicine, National Taiwan University, Taipei, Taiwan myopic eyes in their study had PVAs. Muraoka et al. [3] used the term paravascular inner retinal defect (PIRD) to describe some specific type of PVA lesions. They showed that these changes may be associated with corresponding visual field defect. In both studies, however, there was no mention about full-thickness retinal defects. In addition to high myopia, eyes with ERM also frequently have PVAs, showing similar lesions as those in highly myopic eyes [4]. In our previous study [5], we found that cases with PVAs had significantly greater central macular thickness; the thicker the macula, the more severe the PVA complex. Again, no full thickness macular defect was observed in these cases.

Retinal detachment (RD) with posterior breaks in highly myopic eyes has been reported in previous studies [6-8]. However, whether the breaks were related to PVAs was never reported. In the present study, we presented a case series of RD that developed secondary to PVA-related fullthickness retinal breaks. The common and specific clinical features were explored, and the effect of management on visual outcomes of these cases were investigated. 


\section{Patients and methods}

From July 2015 to June 2017, consecutive cases of RD caused by PVA-related breaks were retrospectively collected. The diagnostic criteria for PVA-related breaks included the presence of one or more full-thickness breaks in the presence of other PVAs in the vicinity, or the detection of multiple paravascular breaks adjacent to each other with or without other PVA lesions along the same vessel. Paravascular breaks associated with peripheral lattice degeneration or fibrovascular tufts from retinal vascular diseases were excluded. The study was approved by the Institutional Review Board of National Taiwan University Hospital.

\section{Data collection}

Before surgery, each patient underwent ophthalmologic examinations including best-corrected visual acuity (BCVA), refraction status (Auto Refractometer KR-8800; Topcon Corporation, Tokyo, Japan), indirect fundoscopy, and optical coherence tomography (OCT) (Cirrus HD-OCT 400 and Stratus OCT 3000, Carl Zeiss Meditec, Dublin, CA, USA; Carl Zeiss Meditec, Dublin, CA; RTVue OCT RT 100, Optovue, Fremont, CA, USA). During the operation, the numbers and positions of the retinal breaks were recorded. The severity of RD was recorded as limited to the posterior pole or beyond the equator. Follow-up examinations after surgery included BCVA, indirect fundoscopy, and OCT at least once every 3 months. OCT features were obtained by multiple scans centered on the fovea. For the retinal break(s) that were found in the posterior pole, OCT through the break(s) was also carried out. Axial length was measured by an ultrasound biometry (PAC Scan 300AP; Sonomed Inc, Lake Success, NY) postoperatively when the retina was well attached. All cases were followed up for at least 6 months after surgery.

\section{Surgical techniques}

A 25-gauged vitrectomy system was set up for all the cases. After core vitrectomy, removal of the posterior hyaloid and ERM were performed. Breaks outside the arcade or at the arcade margin were treated with endolaser after fluid-air exchange; breaks close to the fovea or adjacent to severe choroiretinal atrophy were treated with either inverted internal limiting membrane (ILM) insertion or multiple free ILM flap insertion. Both techniques have been described previously. In short, for inverted ILM flap insertion, a ringshaped ILM island measured about 1.5-3 disc diameters centered on the break was preserved during the procedure of ILM peeling; the ILM flap anchored on the edge of the break was inverted and inserted into the hole using microforceps with the tips closed [9]. For multiple free ILM flap insertion, free ILM flaps torn from the retinal surface were saved on a sponge. Viscoat ${ }^{\circledR}$ was injected into and around the break first to capture the ILM flaps. A piece of previously obtained free ILM flap was picked up with microforceps and was released above the retinal surface; then the microforceps with closed tips was used to nudge the free ILM tissue into the break. The procedure was repeated for the insertion of the second, the third, and/or the fourth piece of the free ILM flap. The inserted ILM flaps supported as well checked one another so they would not float up or loss to the subretinal space. After inverted ILM flap or multiple ILM flap insertion, Viscoat ${ }^{\circledR}$ was reapply on top of the macular hole to further stabilize the inserted tissue [10]. If the detachment extended beyond the equator, another iatrogenic retinal break at temporal upper retina was made for subretinal fluid drainage; care was taken not to disturb the posterior retina during fluid-air exchange to minimize the possibility of ILM flaps' dislodgment. If the possibility of recurrent retinal detachment had been judged high, such as when peripheral strong vitreoretinal adhesion had been difficult to separate, multiple breaks in different quadrants existed, or only faint laser patches surrounding the break could be obtained because of chorioretinal atrophy, silicone oil would be used.

\section{Statistical Analysis}

BCVA was converted to the logarithm of the minimum angle of resolution ( $\operatorname{logMAR})$ for analysis. Wilcoxon signed-rank test was used to compare the preoperative and postoperative BCVA. Spearman's correlation analysis was used to evaluate the correlation between BCVA and OCT characteristics, and multiple linear regression models were used for multivariable analysis. A $P$ value $<0.05$ was considered statistically significant. SAS 9.4 software (SAS Institute Inc., Cary, NC) was used for all statistical analyses.

\section{Results}

\section{Baseline characteristics}

A total of eight eyes from eight patients were collected in this study. There were four men and four women. The mean age was $59.9 \pm 14.4$ years, with seven patients ranging from 53 to 73 years and one patient at 29 years of age. All eyes were highly myopic, some with an extremely long axial length $(>30 \mathrm{~mm})$. The axial length ranged from 27.51 to $36.52 \mathrm{~mm}$ (mean: $31.31 \pm 2.86 \mathrm{~mm}$ ). Two of them had multiple retinal breaks associated with PVA lesions, four had a single break with associated nearby PVA, and two had multiple paravascular retinal breaks along the same 
vessels. All breaks were characterized as round or ovalshaped holes. Only three eyes $(37.5 \%)$ had their breaks located over patchy chorioretinal atrophy. As for the location, two cases had the breaks outside the arcades, five had their breaks within the arcades, and one had a break within the arcade as well as two breaks outside the arcade. For the six eyes with retinal breaks within the arcade, four were at the arcade margins and two at the perifoveal areas. The areas of RD extended beyond the equator in six eyes and were limited in the posterior pole in two eyes.

\section{Intraoperative findings}

During the operation, traction from ERM and/or posterior hyaloid was noticed in all eyes, and all of them had removal of the posterior hyaloid and ERM. Peeling of internal limiting membrane (ILM) was performed in six eyes with break (s) within the arcades; among which inverted ILM flap insertions were performed in two eyes with a perifoveal break and multiple free ILM flaps insertion was performed in another eye with 2 breaks at arcade margin. The breaks were treated with endolaser in five eyes without ILM insertion. At the end of surgery, $\mathrm{C}_{3} \mathrm{~F}_{8}$ infusion was given in five eyes and silicone oil infusion was given in three.

\section{Postoperative anatomical and visual outcomes}

All eyes achieved retinal attachment after one operation. The mean follow-up period was $14.6 \pm 10.3$ months (6 to 38 months). The logMAR of BCVA improved from $0.06 \pm$ 0.10 at baseline to $0.19 \pm 0.22$ at the final visit $(P=0.016)$. All but one eye had improved vision after the surgery, and the one eye which maintained the same vision showed complete disappearance of visual field defect. The baseline characteristics, intraoperative findings, and postoperative visual acuity of all patients are summarized in Table 1.

\section{Representative case reports}

Case 1: A 29 -year-old man with single functioning right eye had had RD and received scleral buckling with the retina reattached postoperatively in the right eye ten years ago. On his visit, he complained of floaters and visual field defect for 3 days prior to the visit. Fundoscopy showed a macula-sparing RD from 12 to 5 o'clock area, with multiple large holes along a superior branch of central retinal vein starting from 3 disc diameters from disc to the periphery (Fig. 1a). Vitreous condensation at multiple areas could be seen. The detached area then progressed rapidly to a macula-off status within several days and the BCVA dropped to 0.05. During the surgery, ERM in the macular area was noted. Retinal breaks with operculum were also noticed (Fig. 1b), indicating previous vitreoretinal traction.

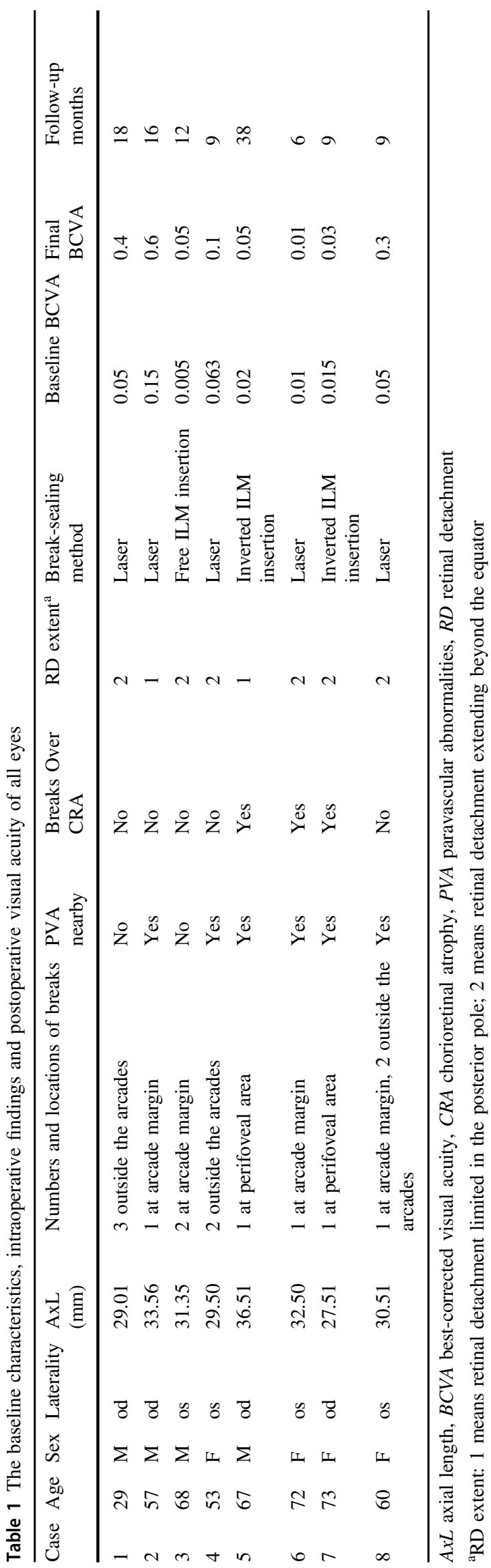


Fig. 1 a Color fundus photography of the right eye of a 29-year-old man showed retinal detachment with multiple paravascular breaks (arrows) along a superior branch of central retinal vein.

b Intraoperatively, an operculum above the retinal break along with vitreous traction to the retina was seen. c After the operation, the retina was reattached. Optical coherence tomography showed a sealed retinal break (arrows) and another branch of retinal vein tenting out from the retinal surface (arrowheads)
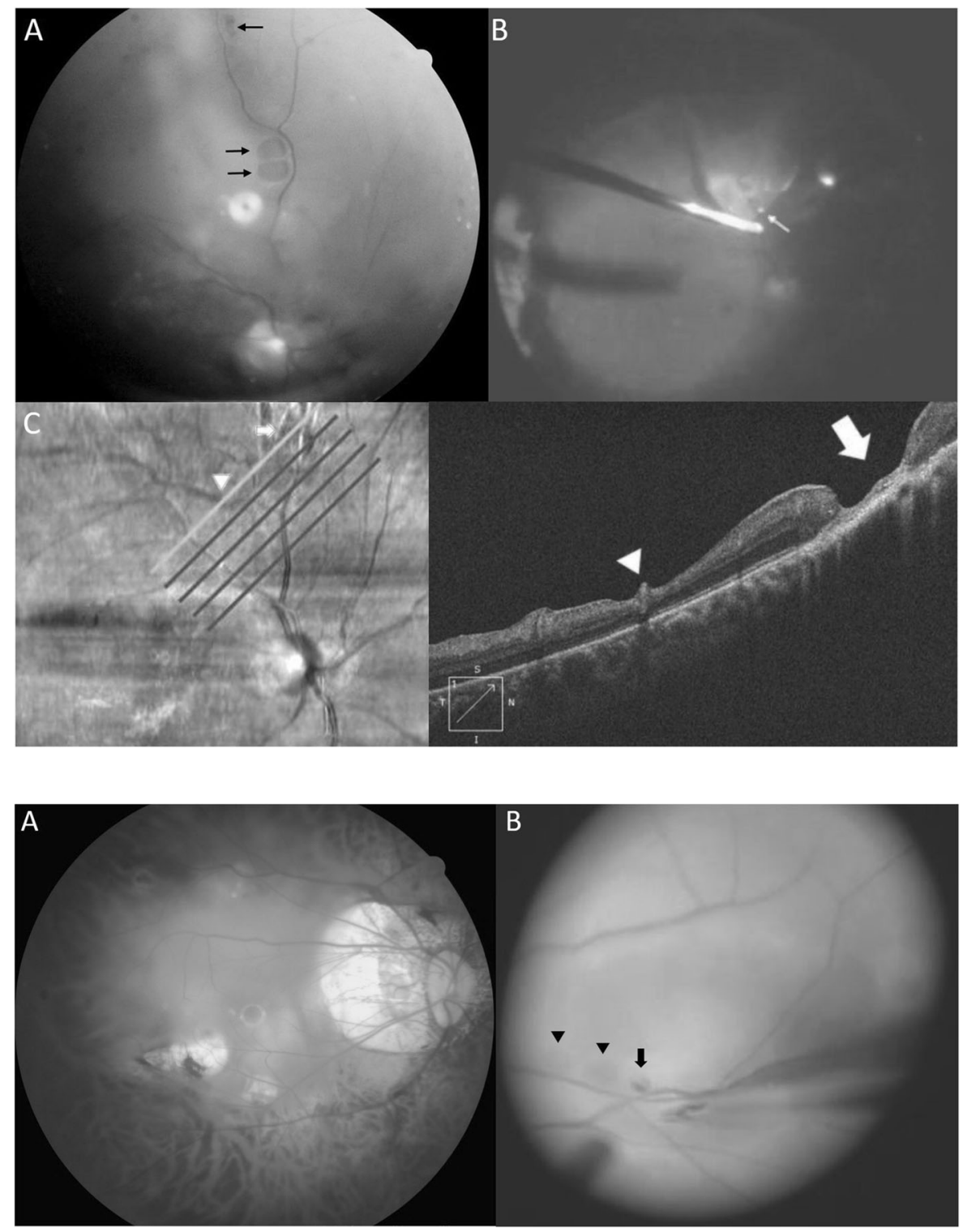

Fig. 2 a Color fundus

photography of the right eye of a 55-year-old man showed retinal detachment of the posterior pole. No definite retinal break was noted. b Intraoperatively, one retinal break (arrow) was noted near the intersection of a retinal artery and a retinal vein in the superior temporal arcade.

Several paravascular abnormalities (arrowheads) were also noted along the retinal artery
Peeling of ERM and ILM in the macular area were performed, and endolaser was applied around the breaks. After the surgery the retina became reattached (Fig. 1c), and the BCVA improved to 0.4 at 18 months postoperatively.

Case 2: A 55-year-old man suffered from progressive decrease in VA in both eyes due to myopic maculopathy for years. On his presentation, he complained of central blurred vision in his right eye. Visual examination showed that the BCVA was 0.15, and fundoscopy and OCT showed a tessellated fundus with patchy chorioretinal atrophy and RD involving the posterior pole. However, no definite break or macular hole was seen (Fig. 2a). During the operation, one full-thickness hole and several lamellar holes were noted along one vessel within the superior arcade (Fig. 2b). Internal drainage through the break and endolaser around the break were performed. The retina was reattached after the surgery, and the BCVA improved to 0.6 sixteen months later.

Case 3: A 68-year-old man with high myopia in both eyes complained of progressive VF defect for 4 to 5 days in the left eye. Previous examinations that took place 5 years ago had shown macular schisis with foveal detachment and ERM. On initial examination, the BCVA was counting fingers at $10 \mathrm{~cm}$. Dense nuclear sclerosis was noted, and fundus examination showed patchy chorioretinal atrophy and retinal detachment involving macula and the lower periphery. Two retinal holes between two vessels in the lower temporal arcade were detected (Fig. 3a). Phacoemulsification with posterior chamber intraocular lens implantation was done followed by vitrectomy with ERM removal and ILM peeling. The holes were found between two chorioretinal atrophic patches (Fig. 3b). Multiple free 
Fig. 3 a Color fundus photography of the left eye of a 68-year-old man showed retinal detachment of the posterior pole. Optical coherence tomography revealed two breaks near the inferior temporal arcade. b During the operation, two retinal holes were noted (arrow indicating one of them).

Multiple free internal limiting membrane (ILM) flaps had been inserted into one of the hole after ILM peeling (arrowhead).

c Postoperatively, optical coherence tomography revealed that the inserted ILM tissues incorporated within the retina and also protruded outside the retinal surface (arrow)

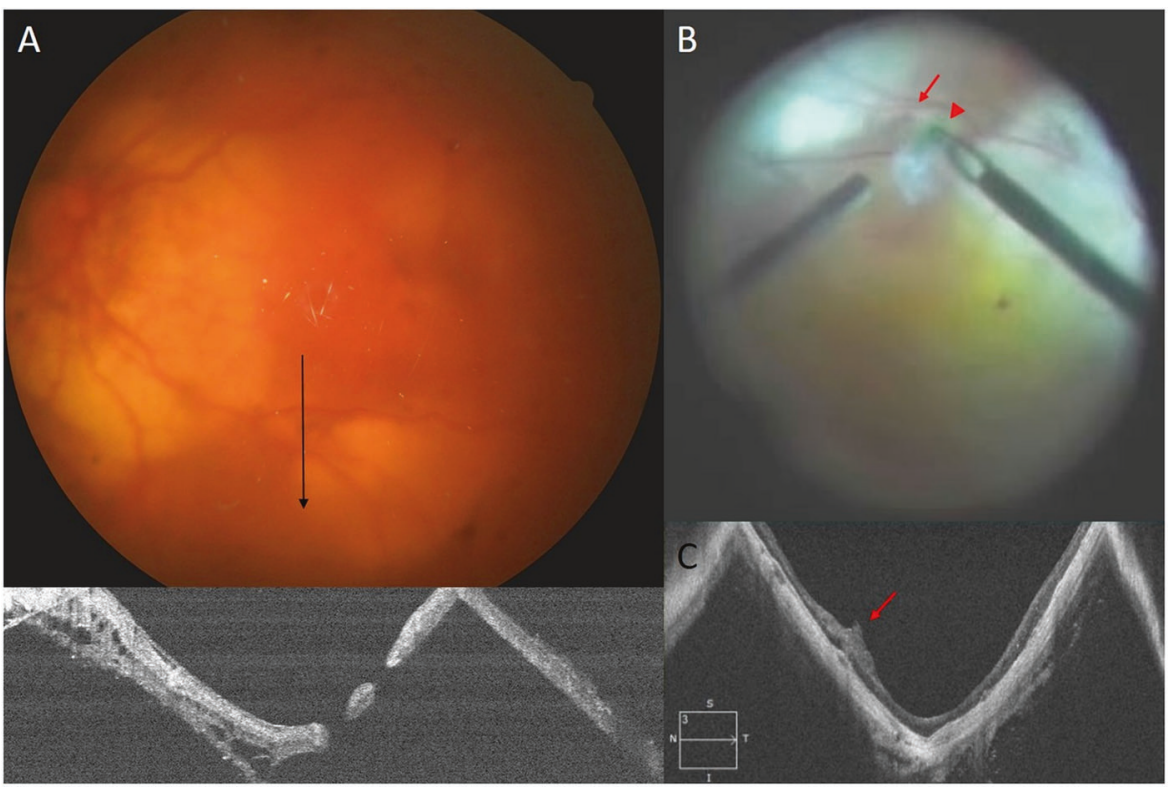

ILM flaps insertion into each hole was performed, and fluidgas exchange was done through another paramacular iatrogenic break. The retina was reattached after the surgery. The inserted ILM flaps could be seen within the retina and protruding above the retinal surface in the lower arcade (Fig. 3c). The BCVA improved to 0.05 at 12 months postoperatively.

Case 4: This was a 53-year-old woman with bilateral high myopia. Her right eye had had persistent macula-off RD under silicone oil tamponade after a previously failed surgery. Her left eye had had a large central chorioretinal atrophic patch with macular scar. The BCVA had maintained 0.03 in the right eye and 0.1 in the left eye for a long time. This time she noticed visual field defect at the nasal side of her left eye for several days, and the BCVA dropped to 0.063 at presentation. Ophthalmological examination revealed RD from 10 to $7 \mathrm{o}^{\prime}$ clock area with the macula attached because of the chorioretinal scar (Fig. 4a), while no apparent breaks could be detected before surgery. Intraoperatively, retinal holes could be observed at two sites outside of arcade: one at the temporal midperiphery and the other along one superior branch of the central retinal vein. Other PVA lesions could also be seen adjacent to the holes in both places (Fig. 4b). Besides, vitreous adhesion to the vessel could be seen along the superior branch retinal vein where the retinal break and PVA were located. Fluid-gas exchange with internal drainage through the temporal break was performed, and endolaser was applied around the breaks and the adjacent PVA at the temporal and the superior midperiphery (Fig. 4c). The retina became attached after the surgery; the BCVA recovered to 0.1 with disappearance of the visual field defect at 9 months after the surgery.

\section{Discussion}

In this small case series, we described the clinical features and surgical outcomes of RD from PVA-related breaks. Six of the eight cases had accompanying PVAs near the breaks; as to the other two cases without PVAs nearby, there were multiple paravascular breaks along the same retinal vessel, which was presumed to come from previous PVAs. About the formation of PVA, we propose that the key factor is the tenting of retinal vessels, which causes stretching and splitting between the inner and outer layers of the paravascular retinal tissues. This abnormal paravascular tissue configuration in turn may cause the formation of retinal cysts, retinoschisis and lamellar retinal hole. PVAs have been described in highly myopic eyes or eyes with ERM. In eyes with high myopia, vessel tenting may be caused by vitreoretinal traction along the vessels or thinning of paravascular retinal tissues secondary to eyeball elongation. Shimada et al. [1] have reported that the presence of PVAs in highly myopic eyes implies eyeball elongation and vitreous traction, which may predispose to the development of macular retinoschisis. As to the PVAs associated with ERM, our previous study [5] showed that central macular thickening due to the traction from ERM may cause paravascular retinal thinning, and the vessels become tented up relative to the surrounding tissue. However, no PVAassociated full-thickness retinal breaks with or without RD have been reported so far. In this study, we specifically aimed at RD from retinal breaks associated with PVAs according to each of the two criteria: first, the observation of other PVA lesions along the vessels adjacent to the breaks; or second, the presence of multiple breaks along the same vessels. These changes were consistent with the characteristics of PVA, in which clusters of lesions of different severity developed along 
Fig. 4 a Color fundus photography of the left eye of a 53-year-old woman showed retinal detachment with the macula sparing due to chorioretinal scar. b During the operation, one break was noted at the temporal midperiphery and the other along a superior branch of the central retinal vein (arrows). Other PVAs could be seen adjacent to the breaks (arrowheads). c Endolaser was applied around the breaks and the adjacent PVA after fluid-air exchange

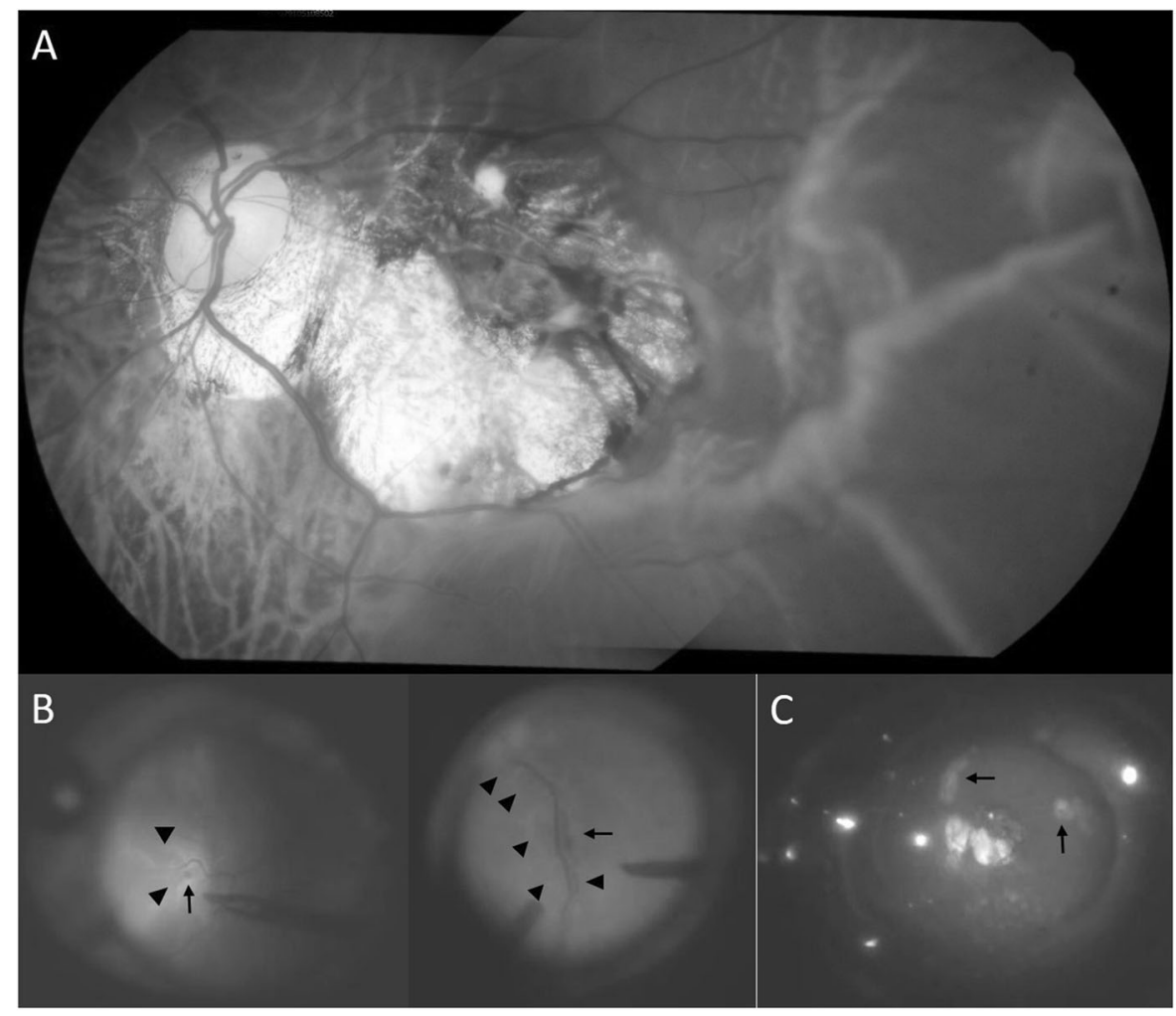

the same vessels. We found that all eyes in the present study were highly myopic with axial lengths of 27.5 to $36.5 \mathrm{~mm}$. It has been proposed that sclerotic modifications and rigidity of retinal vessels in highly myopic eyes may provoke inward tractions, and the concomitant tangential contraction on the contiguous retina may be another relevant factor in the formation of paravascular linear retinal breaks [11, 12]. According to the finding of the present study, we propose that in highly myopic eyes, either the retina is under more traction and stress or the paravascular retinal tissues are more vulnerable to traction, comparing with eyes with only ERM.

In highly myopic eyes, RD caused by posterior breaks other than macular hole has been reported before (Table 2). Benson and Tasman [13] first reported three cases of rhegmatogenous RD caused by paravascular vitreoretinal traction. Dinah et al. [7] reported 7 cases of juxtapapillary microholes in high myopic eyes associated with type 3 posterior staphyloma. In all these 7 cases, there was a single hole located within the peripapillary chorioretinal atrophic area, and shallow and stable RD developed shortly after the onset of posterior vitreous detachment. Dugas et al. [14] studied the cases of RD in highly myopic eyes with axial length $>30 \mathrm{~mm}$ and found posterior paravascular breaks in 11 out of the 67 cases. Chen et al. [6] reported 10 cases of RD due to posterior paravascular breaks in pathological myopia. In their case series, the breaks were all linear-shaped without operculum, located along the posterior vascular arcades, at the edge of posterior staphyloma, and over the areas of patchy chorioretinal atrophy. The mechanisms of the formation of retinal breaks proposed in these papers include vitreous traction, vessel induced inward traction, and the thin retina in the areas of patchy chorioretinal atrophy. Many of these breaks were difficult to see before surgery. Although the description and images presented by Chen et al. [6] did resemble some of our cases, the authors did not relate the breaks with PVAs. Rizzo et al. [8] recently reported 3 cases of $\mathrm{RD}$ due to paravascular or juxtapapillary breaks in pathological myopia. In their cases, all breaks were also located over patchy chorioretinal atrophy. None of these previous studies has mentioned about the associations between PVAs and RD.

Compared to the cases with RD and posterior breaks presented in the above-mentioned studies, the clinical features of the cases in this study were somewhat different from those previously reported. We found that multiple breaks were possible, and the breaks were mainly round or oval in shape. The shape was consistent with the postulation that they developed from lamellar holes, which in turn came from retinal cysts after the inner lining rupture. In addition, unlike described in reports by Chen et al. [6] and Rizzo et al. [8], some of the breaks in our series were locate inside the arcade and were not necessarily located over areas of patchy chorioretinal atrophy or at the edge of staphyloma. In fact, all posterior breaks in our series were within the staphyloma. Furthermore, other PVA lesions may be seen adjacent to the full-thickness breaks, and vitreous traction was noted on adjacent vessels as well as on the break margin. Finally, as 


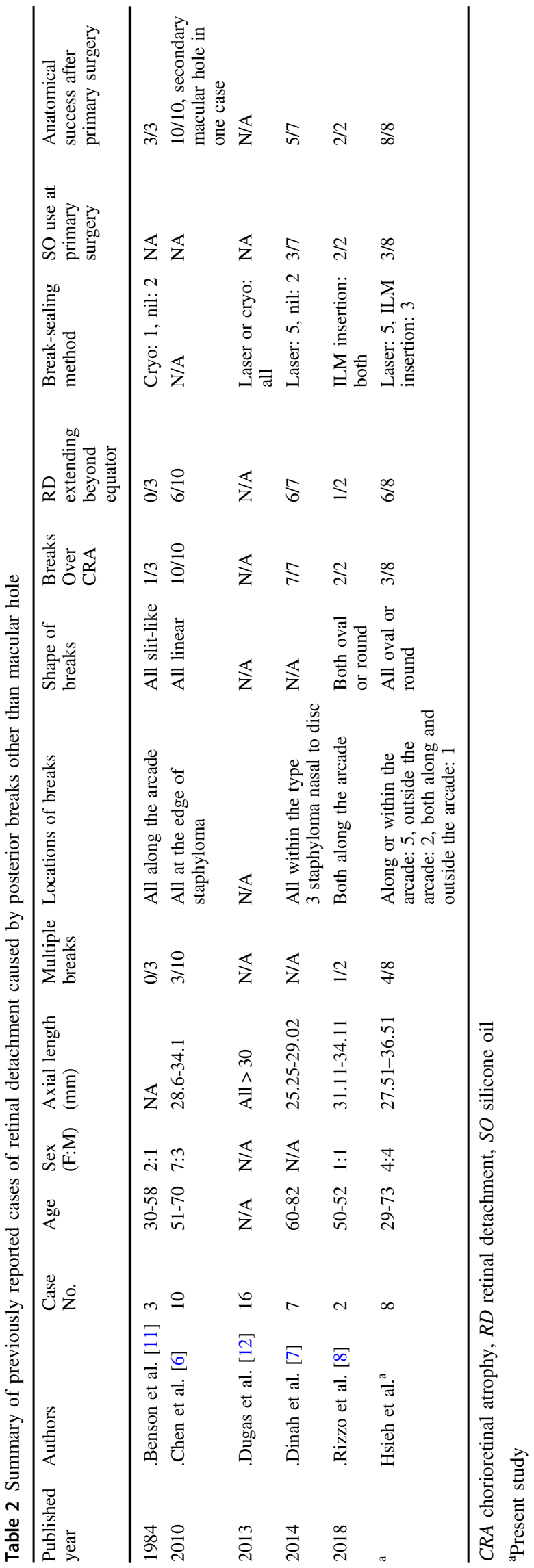

three of our cases showed, breaks associated with PVAs may also be seen in midperiphery outside the staphyloma. The presence of multiple breaks and other PVA lesions along the same vessels strongly indicated that the breaks were related to PVAs. Thus, we propose that PVA may be a mechanism of paravascular breaks in highly myopic eyes, and PVA-related $\mathrm{RD}$ can also have breaks outside the arcade or staphyloma. In those cases suffering from high myopia with posterior RD but no macular hole, one should search for paravascular breaks and make certain to remove all tangential traction form ERM/ ILM in the macular area as well as vitreous traction on the vessels and the breaks.

In previous reports concerning posterior breaks with $\mathrm{RD}$ in high myopia, the methods of treatment generally consisted of vitrectomy, ERM and ILM peeling, with gas or silicone oil tamponade. Although endolaser around the breaks have been mentioned, the underlying chorioretinal atrophy may prevent proper coagulation reaction. In the present study, we suggested if the breaks were close to the foveal center, that is, within 2DD from the center, they should be treated as macular holeassociated RD. The technique of inverted ILM flap or free ILM flap insertion should be performed [9, 10, 15]. As for the breaks that were along the major vessels or away from the center, peeling of posterior hyaloid/EMR/ILM in the macular area should also be performed to release traction on the macular area; drainage from the breaks and laser around the breaks can be done as treating a case of $\mathrm{RD}$ with peripheral breaks. If proper intake of laser energy is in doubt because of the underlying chorioretinal atrophy, inverted ILM flap or free ILM flap insertion may also be applied to ensure hole closure. Rizzo et al. [8] had reported 3 cases of RD due to paravascular or juxtapapillary breaks over patchy chorioretinal atrophy that were successfully treated with autologous ILM transplantation with either free ILM or inverted ILM flaps. Because the traction has been released by surgery, other less severe PVA lesions may not require treatment. However, if the break is away from the posterior pole, other adjacent PVA lesions may be treated with laser to avoid further break formation.

There are still other challenges when managing such cases. As mentioned before, the retinal break could not be identified preoperatively in many cases; it is important to seek the resulting break(s) as well as the associating PVA. Because these eyes are all highly myopic, the instrument tips may not reach the posterior wall in those with extremely long axial lengths. In such situation, the cannula must be removed or even a longer microforceps must be used. However, in this study, because the retina was elevated, the instrument could access to the retinal surface without difficulty. It is also frequent for highly myopic eyes to have residual vitreous cortex adhering to the retinal surface after posterior vitreous detachment, which would increase the difficulty in ILM peeling. Besides, the retina is usually mobile and the risk of iatrogenic retinal 
breaks is relatively high, so less aspiration force and a faster cutting rate should be used. A mild laser energy should be used initially, or else there is a high chance of sieve-like retinal break formation due to the laser burns. However, it would be difficult to titrate the laser energy due to the deficient melanin in retinal pigment epithelium and choroid. Furthermore, care should be taken to avoid eyeball collapsing during fluid-gas exchange as there is a higher risk of suprachoroidal hemorrhage in highly myopic eyes; using valved cannulas may help reduce the incidence of intraocular pressure loss.

In summary, although our case number is limited, we found that full-thickness retinal breaks may be a complication of PVAs. It may cause RD particularly in highly myopic eyes. The condition may be treated as RD caused by peripheral breaks or as macular hole-associated RD depending on the location of the breaks. ERM/ILM peeling should be performed to release all possible traction. Future studies of larger case series are required to confirm our observations.

\section{Summary}

\section{What was known before}

- Paravascular abnormalities (PVAs) including retinal cysts, microfolds, lamellar retinal hole and retinoschisis including detachment of inner internal limiting membrane can be seen in highly myopic eyes or in eyes with epiretinal membrane. Full-thickness retinal hole has never been linked to PVAs before.

- Retinal detachment (RD) with paravascular breaks over patchy chorioretinal atrophy at the posterior pole may happen in highly myopic eyes.

\section{What this study adds}

- PVAs may evolve into full-thickness retinal breaks and then result in a distinct type of RD in highly myopic eyes.

- PVAs and the associated breaks should be searched in RD without macular hole or obvious breaks in highly myopic eyes. Such breaks may or may not locate over patchy chorioretinal atrophy.

- RD from PVA-associated breaks may be treated successfully as RD caused by peripheral breaks or as macular hole associated RD depending on the location of the breaks.

Acknowledgements English editing was provided by the Department of Medical Research at National Taiwan University Hospital.

\section{Compliance with ethical standards}

Conflict of interest The authors declare that they have no conflict of interest.

\section{References}

1. Shimada N, Ohno-Matsui K, Nishimuta A, Moriyama M, Yoshida $\mathrm{T}$, Tokoro T, et al. Detection of paravascular lamellar holes and other paravascular abnormalities by optical coherence tomography in eyes with high myopia. Ophthalmology. 2008;115:708-17.

2. Li T, Wang X, Zhou Y, Feng T, Xiao M, Wang F, et al. Paravascular abnormalities observed by spectral domain optical coherence tomography are risk factors for retinoschisis in eyes with high myopia. Acta Ophthalmol. 2017;96:e515-23.

3. Muraoka Y, Tsujikawa A, Hata M, Yamashiro K, Ellabban AA, Takahashi A, et al. Paravascular inner retinal defect associated with high myopia or epiretinal membrane. JAMA Ophthalmol. 2015;133:413-20.

4. Miyoshi Y, Tsujikawa A, Manabe S, Nakano Y, Fujita T, Shiragami $\mathrm{C}$, et al. Prevalence, characteristics, and pathogenesis of paravascular inner retinal defects associated with epiretinal membranes. Graefes Arch Clin Exp Ophthalmol. 2016;254:1941-9.

5. Liu HY, Hsieh YT, Yang CM. Paravascular abnormalities in eyes with idiopathic epiretinal membrane. Graefes Arch Clin Exp Ophthalmol. 2016;254:1723-9.

6. Chen L, Wang K, Esmaili DD, Xu G. Rhegmatogenous retinal detachment due to paravascular linear retinal breaks over patchy chorioretinal atrophy in pathologic myopia. Arch Ophthalmol. 2010;128:1551-4.

7. Dinah CB, Vaideanu-Collins D, Steel DH. Progressive retinal detachment secondary to juxtapapillary microholes in association with type 3 posterior staphylomas. Clin Ophthalmol. 2014; 8:1089-95

8. Rizzo S, Tartaro R, Barca F, Bacherini D, Franco F, Caporossi T. Autologous internal limiting membrane fragment transplantation for rhegmatogenous retinal detachment due to paravascular or juxtapapillary retinal breaks over patchy chorioretinal atrophy in pathologic myopia. Retina. 2018; 38:198-202.

9. Chen SN, Yang CM. Inverted internal limiting membrane insertion for macular hole-associated retinal detachment in high myopia. Am J Ophthalmol. 2016;166:211.

10. Chen SN, Hsieh YT, Yang CM. Multiple free internal limiting membrane flap insertion in the treatment of macular holeassociated retinal detachment in high myopia. Ophthalmologica. 2018;240:143-9.

11. Ikuno Y, Gomi F, Tano Y. Potent retinal arteriolar traction as a possible cause of myopic foveoschisis. Am J Ophthalmol. 2005; 139:462-7.

12. Sayanagi K, Ikuno Y, Gomi F, Tano Y. Retinal vascular microfolds in highly myopic eyes. Am J Ophthalmol. 2005;139:658-63.

13. Benson WE, Tasman W. Rhegmatogenous retinal detachments caused by paravascular vitreoretinal traction. Arch Ophthalmol. 1984;102:669-70.

14. Dugas B, Bron AM, Minoyan G, Aho S, Paul Berrod J, PC-G C. Primary vitrectomy for the treatment of retinal detachment in highly myopic eyes with axial length over $30 \mathrm{~mm}$. Eur J Ophthalmol. 2013;23:564-70.

15. Chen SN, Yang CM. Double Internal Limiting Membrane Insertion for Macular Hole-Associated Retinal Detachment. J Ophthalmol. 2017;2017:3236516. 